\title{
LAS JORNADAS CIENTÍFICAS ESTUDIANTILES UNA VÍA PARA LA PROMOCIÓN DE COMPETENCIAS INVESTIGATIVAS DE LOS ESTUDIANTES \\ DEL ITF.
}

\section{STUDENT SCIENTIFIC SYMPOSIUMS, A WAY TO PROMOTE INVESTIGATIVE SKILLS OF ITF STUDENTS.}

Evelyn De la Llana Pérez, Lcda.

Licenciada en Sociología (Cuba).

Docente del Instituto Tecnológico de Formación Profesional, Administrativa y Comercial,

Ecuador.

evelyn.delallana@formacion.edu.ec

Rafael Félix Bell Rodríguez, MSc.

Master of arts en educacion (Rusia).

Vicerrector Académico del Instituto Tecnológico de Formación Profesional, Administrativa y

Comercial, Ecuador. rafael.bell@formacion.edu.ec

Yoenia Portilla Castell, Ing. Ingeniero Industrial (Cuba). Coordinadora Metodológica del Instituto Tecnológico de Formación Profesional, Administrativa y Comercial, Ecuador. yoenia.portilla@formacion.edu.ec

Srta. Johana Julaysi Bonoso Fernández Estudiante de la Carrera de Administración de Empresas del Instituto Tecnológico de Formación Profesional, Administrativa y Comercial, Ecuador. julaysi.bonoso@formacion.edu.ec

\section{ARTÍCULO DE INVESTIGACIÓN}

Recibido: 28 de agosto de 2018.

Aceptado: 2 de noviembre de 2018. 


\section{RESUMEN}

La formación de competencias investigativas en los estudiantes del Instituto Tecnológico de Formación Profesional, Administrativa y Comercial se concibe implícitamente en el Plan de estudio con actividades interdisciplinarias y transdisciplinarias que se llevan a cabo por los docentes durante el paso de los estudiantes por la institución como parte del proceso enseñanza -aprendizaje. En conjunto con esto se desarrollan actividades como las Jornadas Científicas Estudiantiles que han sido un elemento importante en la motivación de los estudiantes para investigar sobre diversas temáticas. En el presente trabajo se hace una contextualización de la formación de competencias investigativas en los estudiantes del instituto a través del análisis de la participación de los mismos en las cinco Jornadas Científicas Estudiantiles que se han desarrollado hasta la actualidad.

Palabras clave: Aprendizaje, Competencias investigativas, Formación profesional.

\section{ABSTRACT}

The training of research skills in the students of the Technological Institute of Professional, Administrative and Commercial Training is implicitly conceived in the Plan of study with interdisciplinary and transdisciplinary activities that are carried out by the teachers during the passage of the students by the institution as part of the teaching-learning process. In conjunction with this, activities such as the Student Scientific Conferences that have been an important element in the motivation of the students to investigate different topics are developed. In the present work, a contextualization of the training of research skills in the students of the institute is made through the analysis of their participation in the five Student Scientific Workshops that have been developed up to the present.

Keywords: Learning, investigative skills, professional training.

\section{INTRODUCCIÓN}

La adquisición de competencias investigativas en los estudiantes debe considerarse un camino muy significativo no solo para el logro de grandes descubrimientos científicos y tecnológicos, sino también, un camino que favorezca el desarrollo en los estudiantes de habilidades que les permitan apropiarse, interpretar y comprender mejor el mundo en el que habitan para de este modo contribuir a la solución de problemas presentes en los diferentes contextos en los que se desarrollan. Para ello se hace preciso que la investigación se convierta 
en una parte inseparable del proceso formativo de las y los estudiantes.

El Instituto Tecnológico de Formación Profesional, Administrativa y Comercial en los últimos años ha venido desarrollando una serie de actividades curriculares y extracurriculares en función de despertar en los estudiantes el interés por la investigación científica más allá de la mera presentación de un trabajo de titulación como culminación de sus estudios. Como parte de estas actividades se destacan las Jornadas Científicas Estudiantiles que desde su surgimiento en el año 2016 han ido incidiendo en los estudiantes de forma positiva motivándolos progresar en el desarrollo de investigaciones más contextualizadas y constructivas.

\section{REVISIÓN TEÓRICA}

El término competencias viene del latín cum y petere, (RAE, 2001), que significa capacidad para concurrir, coincidir en la dirección. Para Tobón (2006,) las competencias son la capacidad de seguir en un área determinada, suponiendo una situación de comparación directa y situada en un momento determinado. Se ha empleando con tres significaciones: pertenecer, rivalizar y adecuado.

Hernández (2005) se refieren a las competencias como una construcción, resultado de una combinación pertinente de varios recursos, una persona es competente si sabe actuar de manera pertinente en un contexto particular, eligiendo y movilizando un equipamiento doble de recursos personales y redes.

"Una de las fuentes claves de información para la evaluación integral de la calidad de la docencia la constituyen los estudiantes, pues el conocimiento del nivel de satisfacción con la educación que reciben y las condiciones en las que la misma transcurre, aporta una información indispensable para la identificación de las fortalezas y debilidades presentes en este proceso a fin de adoptar las medidas y estrategias que garanticen la elevación permanente de la calidad de la educación" (Cadena-Badilla, Mejías Acosta, Vega-Robles, \& Vásquez Quiroga, 2015, p. 67).

Le Boterf (2001) por su parte las define como un saber hacer complejo, resultado de la integración, movilización y adecuación de las capacidades (cognitivas, afectivas y sociales) y los conocimientos. 
Las competencias investigativas buscan la autonomía de la persona y se orientan hacia la autorrealización del sujeto, como vía para la realización de un proyecto vital, que satisfagan las necesidades de la comunidad. Los individuos son concebidos como objeto y sujeto de la transformación. Por lo tanto, este planteamiento de las competencias investigativas tiene como objetivo prioritario el desarrollo y consolidación de la cultura investigativa.

Las definiciones estudiadas, permiten relacionar elementos comunes como idoneidad, conducta organizada, actuación, saber hacer y estructuras mentales, concentradas en la dimensión del conocer- hacer. Para que se ajusten a lo que se aspira en la educación superior, deben abordarse como un tejido multidimensional, solo así se producirá el verdadero cambio educativo, es así como surge el concepto de competencias profesionales. La CEPAL (1992), en su declaración de Chile, considera que los estudiantes universitarios, deben ir más allá del conocimiento de técnicas y herramientas para resolver problemas, y vincularse con lo afectivo, con lo cognoscitivo y lo actuacional, lo cual exige para el sujeto que se precise como competente capacidad de análisis, responsabilidad, compromiso y resistencia a la incertidumbre.

La cultura investigativa es una alternativa de desarrollo y progreso no sólo referido a lo laboral, sino también en el plano social. De ahí la necesidad de fomentarla en el proceso formativo de los futuros profesionales, sobre la base de las competencias investigativas que se vincule motivado por razones de orden personal y social. En el plano personal, el hecho de investigar, supone un ejercicio de madurez, responsabilidad y autogobierno que se muestra mediante un alto grado de autonomía de los sujetos. La asunción de la cultura investigativa desde el punto de vista social, implica conocimiento, manejo y participación en los proyectos sociales. Un saber que llevado a la práctica permite la integración y el intercambio social, básicos para el desarrollo comunitario con base en la acción, el compromiso y la equidad.

El proceso de enseñar e investigar es una actividad compleja que recoge numerosas operaciones. Para lograr un buen aprendizaje se hace necesario que se involucre a los estudiantes en todas las tareas de esta práctica, unido a otra persona con mayor experiencia, los docentes, y en el que se promueva la investigación en diferentes campos de la ciencia. 
Enseñar a investigar según Sánchez (2014) es un proceso complejo y una actividad diversificada. La nueva didáctica de la investigación social y humanística que se propone es, en primer lugar, un proceso complejo, pues en la enseñanza de la investigación concurren numerosas operaciones, no menos densas y relativas a:

1) lo que se enseña al enseñar a investigar, y

2) cómo se enseña a investigar.

Estos dos tipos de operaciones constituyen dos prácticas distintas, la de producir conocimiento nuevo y la de enseñar a producirlo. La primera conforma el oficio de investigador; la segunda, el oficio de pedagogo. El desarrollo del estudio realizado por Sánchez (2014) traza los múltiples perfiles del encuentro en un solo hombre de estos dos quehaceres que, separados, son ya altamente complicados.

Un aprendizaje basado en la formación y desarrollo de competencia según Torres (2002) prepara al estudiante para la vida porque lo ubica en una problemática real, se desarrollan habilidades de comunicación, autoaprendizaje y pensamiento crítico; se aprende a trabajar en equipo e integrar diferentes aspectos del currículo. Además, permite que se articule la teoría con la práctica; favoreciendo en los estudiantes el sentido de responsabilidad y compromiso social. El aprendizaje basado en competencias posibilita que los estudiantes aprendan a tomar decisiones; se adquiere actitud hacia el cambio y la innovación, se aborda el problema de manera total y tanto el docente como el estudiante reconocen que siempre existen posibilidades de aprender.

Dentro de esta concepción pedagógica es de vital importancia comprender que los motivos, intereses, necesidades y actitudes del individuo constituyen componentes importantes, como motores impulsores de la construcción y desarrollo de las competencias.

De igual forma Rizo (2004,) señala la importancia de que los docentes que enseñan investigación, manejen competencias investigativas para transferirlas de forma eficaz a sus estudiantes, plantea que pensar la investigación supone una aproximación a los conocimientos teóricos que fundamenta su praxis investigativa, pero además señala la necesidad de darle un sentido reflexivo y asumirla como un proceso en continua construcción 
y reconstrucción, sugiere enseñar a investigar investigando, desde la práctica, tomando en consideración los niveles pedagógico, epistemológico y comunicativo.

Parafraseando a Zemelman, (2009) se debe trabajar con los educandos en la cultura del pensamiento epistémico. El mismo que está constituido según el autor por el distanciamiento de contenidos elaborados por la teoría, por aquella que ha enmarcado la realidad en un concepto. Por aquella teoría como serie de representaciones, principios, leyes, criterios que apoyan, sustentan o están detrás de nuestras acciones y en las cuales se enmarcan nuestras actividades y planes de vida, social, económicos, culturales, políticos, etc.

Otra mirada al concepto de investigación es adentrarse en una tecnología, aparato o procedimiento y darle alguna aplicación concreta de acuerdo con unos requerimientos; en este sentido correspondería repasar sobre lo planteado por Hernández (2005), quien asevera que algunos sectores han llegado a la conclusión que es necesario desmitificar la investigación, contextualizarla y promoverla en todos los niveles. A pesar de esta diversidad de interpretaciones en constante evolución sigue siendo común que se coloque el status de investigador sólo a aquellas personas de renombre y trayectoria por correr la frontera del conocimiento en campos de interés mundial.

Según Restrepo (1998), la denominada investigación formativa en la educación superior es un tema o problema pedagógico. Aborda, en efecto, la relación docencia-investigación o el papel que puede cumplir la investigación en el aprendizaje de la misma investigación y del conocimiento, problema que se sitúa en el campo de las estrategias de enseñanza y evoca concretamente la docencia investigativa o inductiva y también lo que varios autores han denominado aprendizaje por descubrimientos.

También se debe considerar que la educación es un instrumento privilegiado por cuanto persigue la construcción de un ideal humano mediante estrategias, medios, recursos, etc., con los que se genera la adquisición de valores, creencias, conocimientos, actitudes, saberes, habilidades, entre otros aspectos necesarios para promover el desarrollo de una cultura investigativa, orientada a la consecución del proyecto social aspirado. 
No existe un método único para enseñar a investigar. El método debe ser entendido más bien como la organización estratégica de todas las operaciones que intervienen en la producción científica. El investigador, al formular su problema, planifica y conduce racionalmente sus decisiones teóricas, prácticas, operativas e instrumentales con la intención de encontrar una respuesta a su pregunta.

En línea con lo antes señalado, se produce el estudio y la reflexión acerca de la evaluación integral de la docencia por parte de los estudiantes, en cuyo caso los análisis giran en torno a los principios que han de servir como sustento para el diseño y la aplicación de diferentes estrategias e instrumentos que respondan a los objetivos estratégicos de este tipo de evaluación.

\section{MATERIALES Y MÉTODOS}

El referente empírico de este estudio son los procesos, prácticas y estrategias empleadas en el Instituto Tecnológico de Formación Profesional, Administrativa y Comercial en la formación de competencias investigativas en los estudiantes realizando un análisis cuantitativo y cualitativo de la participación de los mismos en las Jornadas Científicas Estudiantiles. Además, se aplicó una breve encuesta para conocer la satisfacción de los estudiantes con las Jornadas Científicas Estudiantiles; empleando un muestreo probabilístico.

Independientemente de la diversidad de posturas y criterios, es innegable el interés que en la actualidad existe en una parte creciente de los estudiantes por conocer cómo mejorar la calidad de la docencia. En respuesta a ese interés, los cuestionarios ofrecen una vía para valorar sus opiniones y propiciar un mejoramiento de la gestión y el desarrollo de los programas académicos (Cortés, Campos \& Moreno, 2014).

En el presente artículo se realiza un análisis desde el punto de vista cuantitativo y cualitativo de la participación de los estudiantes en las Jornadas Científicas Estudiantiles desde su 
primera edición hasta la actualidad. Este análisis se realizó tomando en cuenta la base de datos del Departamento de Investigación; las que muestran las principales temáticas de investigación y su relación con las líneas de investigación del Instituto Tecnológico de Formación.

Es importante señalar que la primera Jornada Científica Estudiantil se realizó en el año 2016 y en la misma participaron los estudiantes de la Carrera de Marketing. Antes de esta edición la presentación de trabajos donde se mostraron los avances de los estudiantes en el ámbito investigativo se realizó de manera espontánea, organizados por los docentes al interior de las diferentes materias que se impartían en los salones de clase; sin embargo, con esta primera edición de la Jornada Científica Estudiantil interviene el Departamento de Investigación como organizador del evento. Con la realización del evento se logró que estudiantes de diferentes paralelos y horarios de estudio, intercambiaran sobre artículos realizados y temas de interés en la Carrera de Marketing.

Gráfico 1:Ponentes en las JCE

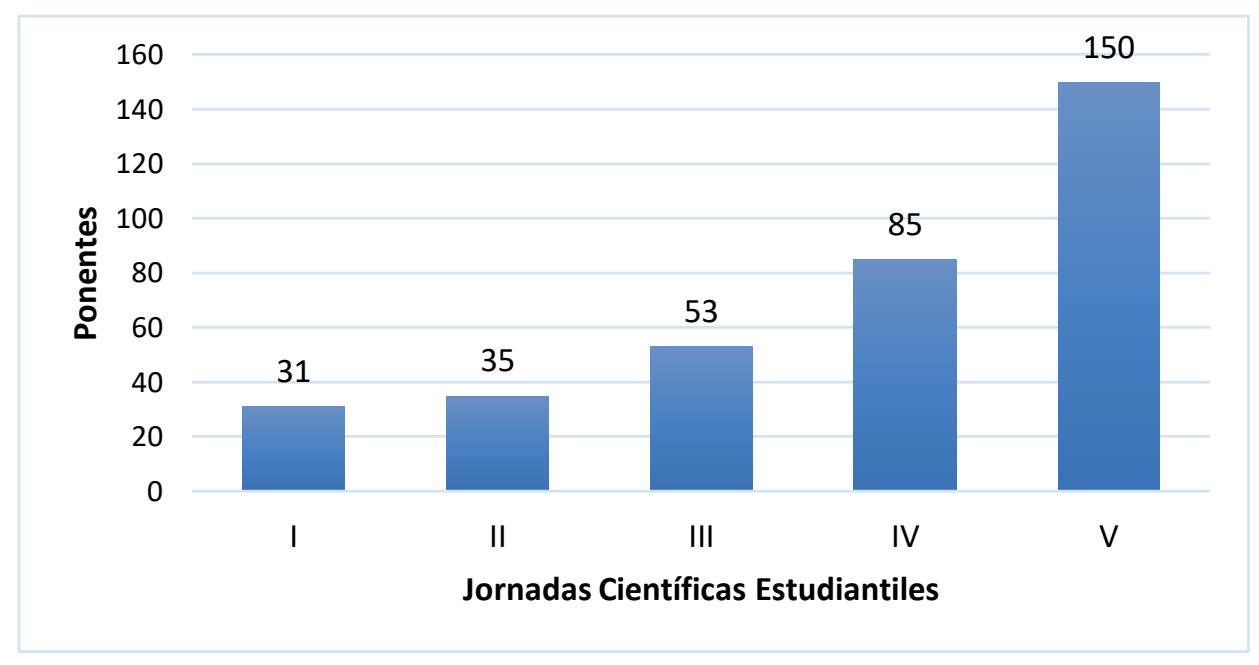

Fuente: Base de datos del Departamento de Investigación del ITF.

En el gráfico se puede observar el aumento y participación de ponentes en las Jornadas Científicas Estudiantiles. Una muestra de cómo los estudiantes se sienten más motivados e 
interesados en participar de este tipo de eventos científicos. Cabe destacar que las primeras tres Jornadas Científicas Estudiantiles se realizaron con estudiantes de una sola carrera y luego de la cuarta se combinaron varias carreras dentro de una misma jornada.

Gráfico 2: Distribución por sexo de los ponentes en las JCE

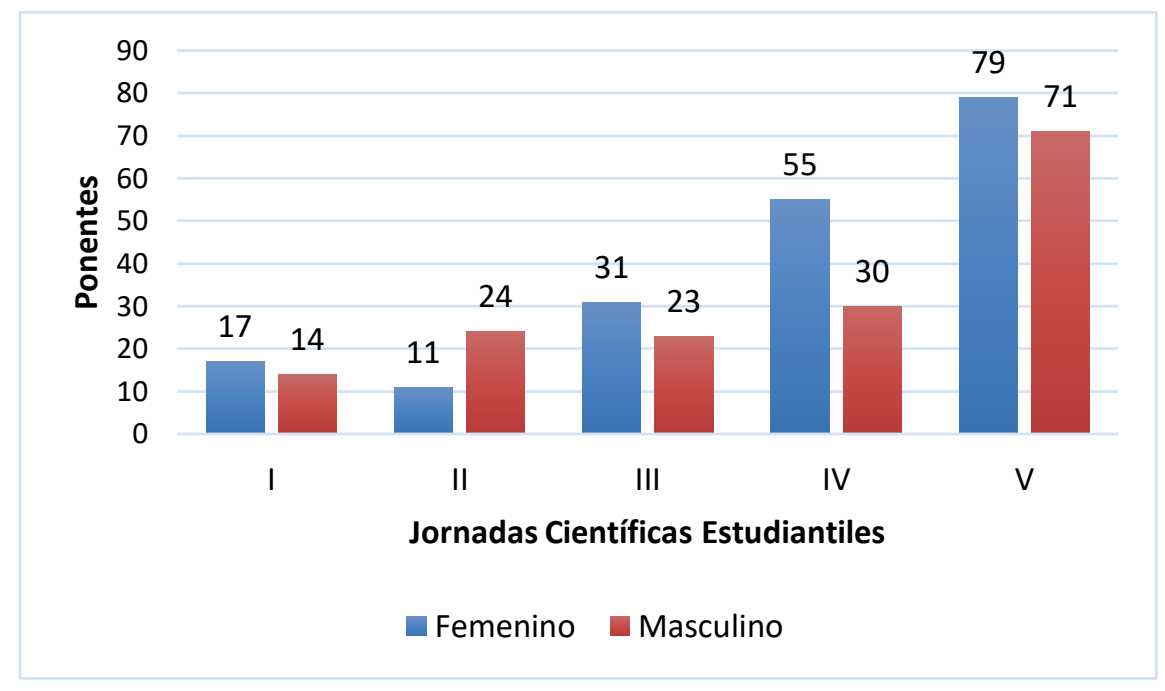

Fuente: Base de datos del Departamento de Investigación del ITF.

Los datos obtenidos de la base de datos del Departamento de Investigación muestran como el sexo femenino ha tenido un protagonismo en la presentación de trabajos investigativos. En todas las Jornadas Científicas Estudiantiles a excepción de la segunda que estuvo dedicada a la Carrera de Diseño Gráfico, las féminas han tenido protagonismo; sin embargo, en la segunda edición se considera que la matrícula que presentaba la Carrera de Diseño Gráfico en el año 2017 con predominio del sexo masculino pudo influir en el comportamiento de este indicador. 
Gráfico 3: Ponentes con discapacidad.

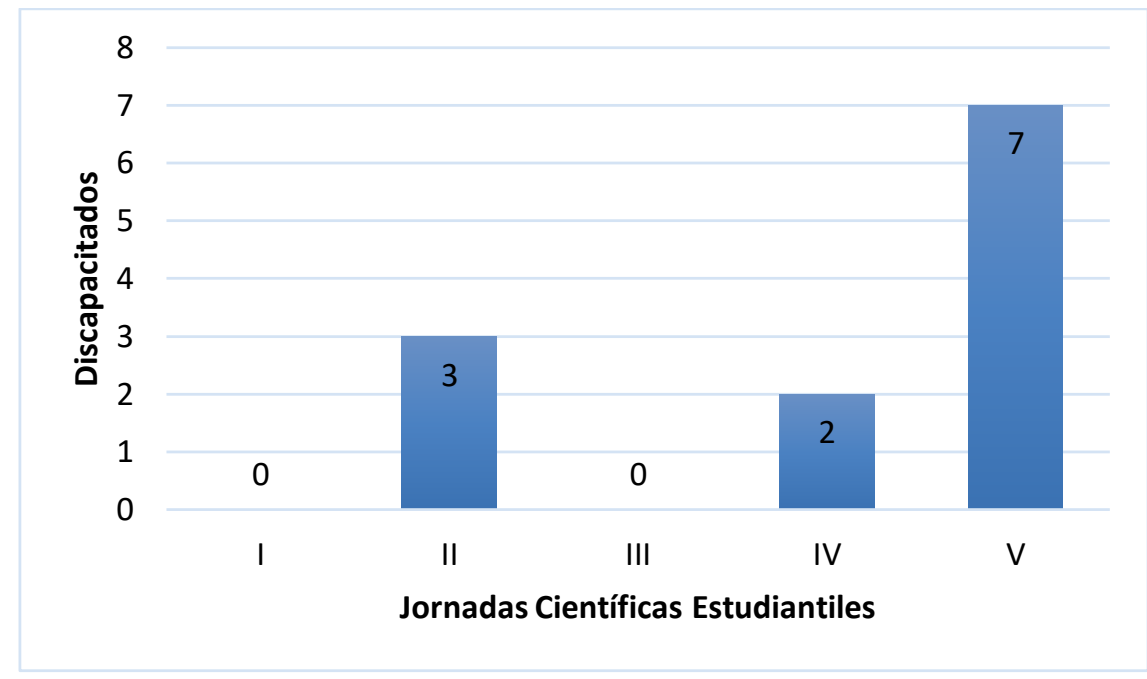

Fuente: Base de datos del Departamento de Investigación del ITF.

Los estudiantes con discapacidad han estado presentes en las Jornada Científicas Estudiantiles. En la segunda jornada participaron tres estudiantes de los diez que componían la matrícula del instituto lo que representó el 30\%. Los estudiantes que se presentaron a la jornada presentan discapacidades físico motoras, intelectual y auditiva. En la primera y en la tercera jornada no participaron estudiantes, sin embargo, en la cuarta participaron dos estudiantes lo que representó el $7 \%$ de los 28 estudiantes con discapacidad que estudian en el Instituto de Formación, cabe destacar que el 100\% de los estudiantes discapacitados de las carreras del horario matutino convocadas para participar en la jornada científica.

La quinta jornada arrojó resultados significativos en cuanto a la participación de los estudiantes con discapacidades especiales, donde se muestra la participación de siete estudiantes lo que equivale al $25 \%$ del total de discapacitados; en relación con los 15 estudiantes convocados para participar fue el $47 \%$. En esta jornada fueron convocadas todas las carreras de la jornada nocturna y adicional a ello los estudiantes de la Carrera de Diseño Gráfico de la jornada matutina. 
Gráfico 4:Cantidad de comisiones por JCE.

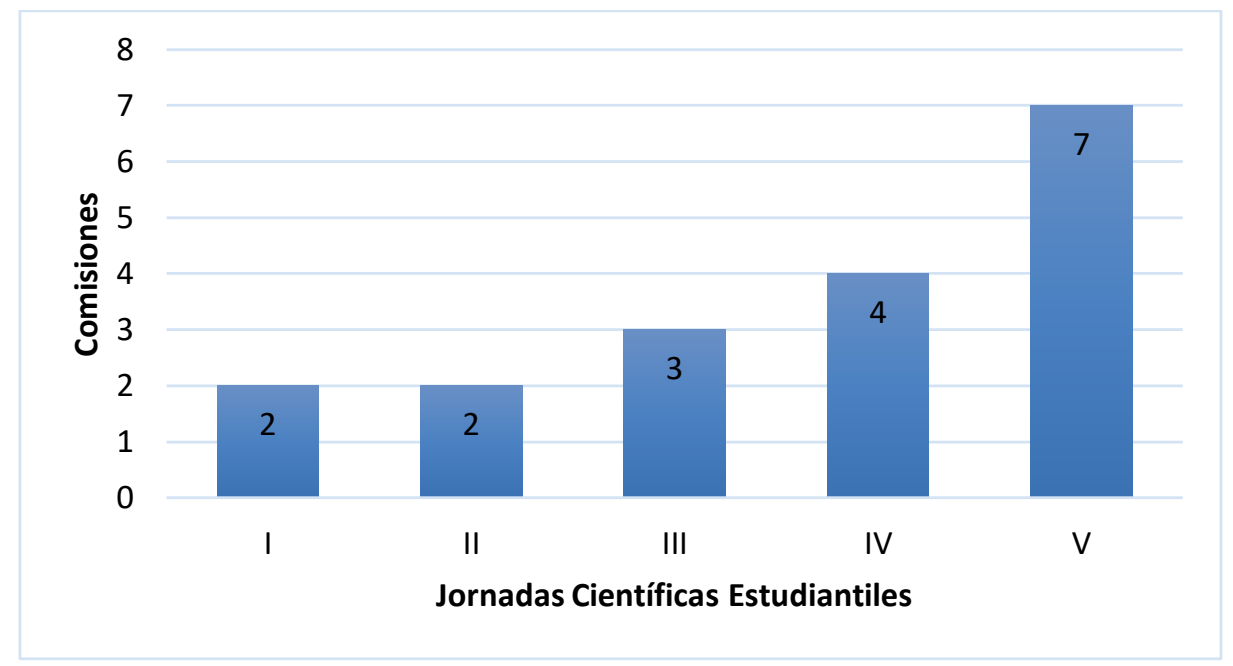

Fuente: Base de datos del Departamento de Investigación del ITF.

El número de comisiones por jornadas han aumentado de forma considerable. En las tres primeras ediciones se convocó una carrera para la realización de las mismas; en el caso de las carreras de Marketing y Diseño Gráfico se organizaron dos comisiones atendiendo a la cantidad de trabajos inscritos para participar. La tercera jornada científica estuvo dedicada a la Carrera de Administración de Empresa para lo cual se organizaron tres comisiones atendiendo al principio de inscripción.

\section{ANÁLISIS DE LOS RESULTADOS}

En la cuarta jornada científica se convocaron las carreras de Administración de Empresa y Contabilidad y Auditoría; en esta última participaron los estudiantes del paralelo DPD 32 de la jornada matutina sin embargo en un solo curso se inscribieron 15 trabajos de los 35 que se presentaron de esta jornada. 
Gráfico 5: Trabajos presentados por JCE.

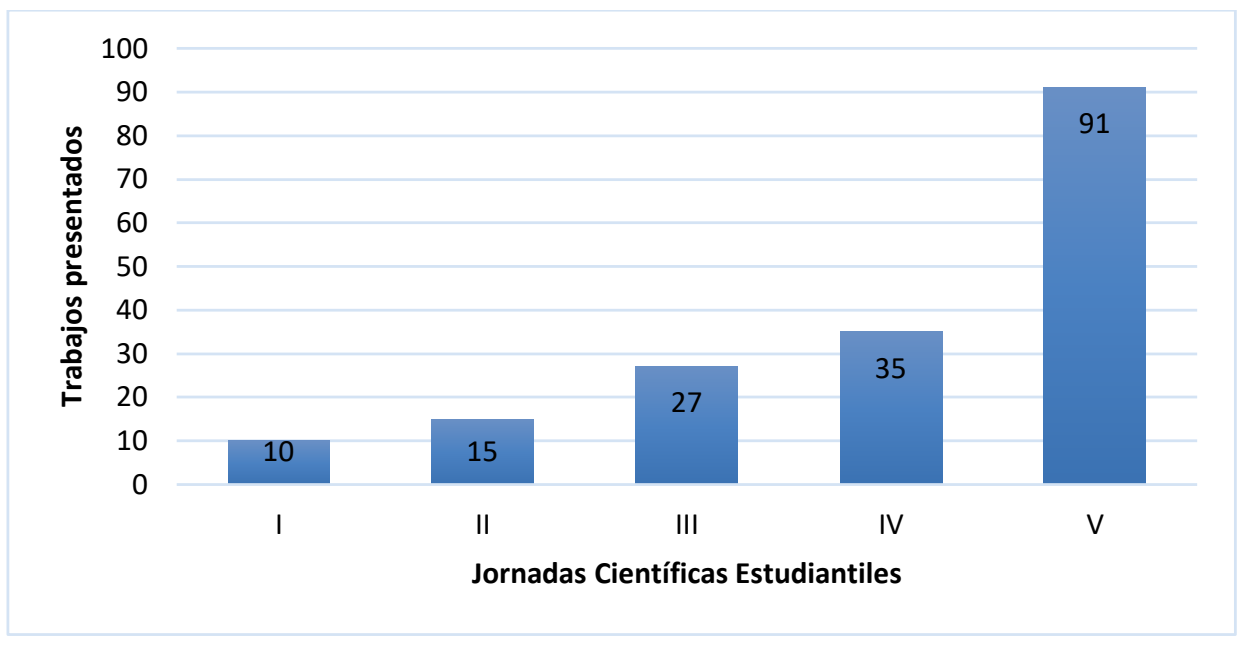

Fuente: Base de datos del Departamento de Investigación del ITF.

El incremento de trabajos presentados por jornadas científicas ha ido aumentando en cada una de las ediciones. Evidenciando un aumento por edición que supera de los 56 trabajos a 91 trabajos participantes destacando la edición anterior.

Tabla 1: Líneas de investigación.

\begin{tabular}{|l|l|}
\hline JCE & Líneas de Investigación \\
\hline I & Comercial y Administrativa \\
\hline II & Artística \\
\hline III & Comercial y Administrativa \\
\hline \multirow{2}{*}{ IV } & Comercial y Administrativa \\
\cline { 2 - 2 } & Educación y Sociedad \\
\hline \multirow{4}{*}{ V } & Comercial y Administrativa \\
\cline { 2 - 3 } & Educación y Sociedad \\
\cline { 2 - 2 } & Artística \\
\hline
\end{tabular}

Fuente: Elaboración propia 
Las líneas principales de investigación del instituto se han abordado en las cinco Jornadas Científicas Estudiantiles. Los trabajos presentados en cuatro de las ediciones se inclinan a la Línea Comercial y Administrativa. Mientras que la Línea de Educación y Sociedad tuvo representatividad en dos de las cinco jornadas realizadas al igual que la Artística.

Tabla 2: Carreras participantes en las JCE.

\begin{tabular}{|l|l|}
\hline JCE & Carreras participantes \\
\hline I & Marketing \\
\hline II & Diseño Gráfico \\
\hline III & Administración de Empresa \\
\hline \multirow{4}{*}{ IV } & Administración de Empresa \\
\cline { 2 - 3 } & Contabilidad y Auditoría \\
\hline \multirow{4}{*}{ V } & Administración de Empresa \\
\cline { 2 - 3 } & Contabilidad y Auditoría \\
\cline { 2 - 3 } & Comercio Exterior \\
\cline { 2 - 3 } & Marketing \\
\cline { 2 - 2 } & Diseño Gráfico \\
\hline
\end{tabular}

Fuente: Elaboración propia

Desde la primera hasta la tercera edición las Jornadas Científicas Estudiantiles se realizaron con la participación de una carrera. A partir de la cuarta edición ya se comienzan a integrar carreras como Administración de Empresa y Contabilidad y Auditoría en el horario matutino. Debido a la motivación que mostraron los estudiantes del horario nocturno ante el conocimiento de la realización de la cuarta jornada, el Departamento de Investigación decidió realizar la quinta edición de la Jornada científica estudiantil en el horario nocturno y con la participación de todas las carreras que asisten en ese horario.

Tabla 3: Líneas temáticas de las JCE.

\begin{tabular}{|l|l|}
\hline JCE & Líneas temáticas \\
\hline I & Mercadotecnia y publicidad \\
\hline \multirow{2}{*}{ II } & Diseño y Comunicación Visual \\
\cline { 2 - 2 } & Elementos visuales y su impacto \\
\hline
\end{tabular}




\begin{tabular}{|c|c|}
\hline \multirow[b]{2}{*}{ III } & Gestión empresarial \\
\hline & $\begin{array}{l}\text { Responsabilidad social, cultura de la calidad y satisfacción del } \\
\text { cliente }\end{array}$ \\
\hline \multirow{4}{*}{ IV } & Gestión empresarial \\
\hline & $\begin{array}{l}\text { Responsabilidad social, cultura de la calidad y satisfacción del } \\
\text { cliente }\end{array}$ \\
\hline & Las Tics y su aplicación en el ámbito empresarial \\
\hline & Emprendimiento e innovación \\
\hline \multirow{7}{*}{ V } & Gestión empresarial \\
\hline & $\begin{array}{l}\text { Responsabilidad social, cultura de la calidad y satisfacción del } \\
\text { cliente }\end{array}$ \\
\hline & Las Tics y su aplicación en el ámbito empresarial \\
\hline & Emprendimiento e innovación \\
\hline & Mercadotecnia y publicidad \\
\hline & Diseño y Comunicación Visual \\
\hline & Elementos visuales y su impacto \\
\hline
\end{tabular}

Fuente: Elaboración propia

Las temáticas de investigación se han ido diversificando al interior de las principales Líneas de Investigación del instituto. En el caso de la Línea Artística se han presentado trabajos relacionados con el Diseño y la Comunicación Visual, así como Elementos Visuales y su impacto. La Línea Comercial y Administrativa se ha visto abordada en cuatro de las cinco jornadas que se han realizado con investigaciones en el campo de la Mercadotecnia y Publicidad y la Gestión Empresarial. La Responsabilidad social, la cultura de la calidad y la satisfacción del cliente han sido abordados desde la Línea de Educación y Sociedad con un énfasis en temas como el clima ético en las empresas y la inclusión en los contextos laborales. Las Tics y su aplicación en el ámbito empresarial han sido abordadas por algunos de los trabajos que se han presentado tanto en la Carrera de Diseño Gráfico como en las de perfil administrativo y comercial.

Las encuestas realizadas se aplicaron con el objetivo de conocer los criterios de los estudiantes respecto a la utilidad de las Jornadas Científicas Estudiantiles realizadas en el año 2018 correspondientes a la cuarta y quinta edición. Se realizó un análisis de las 
percepciones de los estudiantes sobre la calidad de los eventos y la organización de los mismos. Para la realización de la encuesta se empleó un muestreo probabilístico. Considerando una población de 1400 estudiantes se obtuvo una muestra de 302 estudiantes con un nivel de confianza del 95\% y un margen de error del 5\%. A continuación, se muestra la ecuación utilizada para el cálculo del tamaño de la muestra.

Cálculo de la muestra

Donde:

$$
n=\frac{Z_{\alpha}^{2} N p q}{e^{2}(N-1)+Z_{\alpha}^{2} p q}
$$

$\mathrm{n}$ : Tamaño de la muestra

Z: constante que depende del nivel de confianza

$\mathrm{N}$ : tamaño de la población o universo (número total de posibles encuestados)

$\mathrm{p}: \%$ de probabilidad de que ocurra

q: \% de probabilidad de que no ocurra

e: error admisible

En la tabla 4 se muestran los niveles de confianza según el valor de Z. Para el caso de estudio se tomó un valor de 1,96 lo que indica la probabilidad de que los resultados de la investigación sean ciertos en un $95 \%$.

Tabla 4. Nivel de confianza según el valor de Z.

\begin{tabular}{|ll|l|l|l|l|l|l|l|l|}
\hline Valor de Za & 1.28 & 1.65 & 1.69 & 1.75 & 1.81 & 1.88 & 1.96 & 2.58 \\
\hline $\begin{array}{l}\text { Nivel } \\
\text { confianza }\end{array}$ & de & $80 \%$ & $90 \%$ & $91 \%$ & $92 \%$ & $93 \%$ & $94 \%$ & $95 \%$ & $99 \%$ \\
\hline
\end{tabular}

Fuente: Elaboración propia

\subsection{Las Jornadas Científicas Estudiantiles contribuyen a mi formación profesional.}

En el gráfico 6 se puede observar como el $82 \%$ de los estudiantes está muy de acuerdo con que las Jornadas Científicas Estudiantiles lo que contribuyen a su formación profesional. Este resultado muestra el aporte investigativo de los estudiantes y docentes del Instituto de Formación a los resultados de las investigaciones realizadas. 
Gráfico 6: Las Jornada Científicas Estudiantiles contribuyen a mi formación profesional.

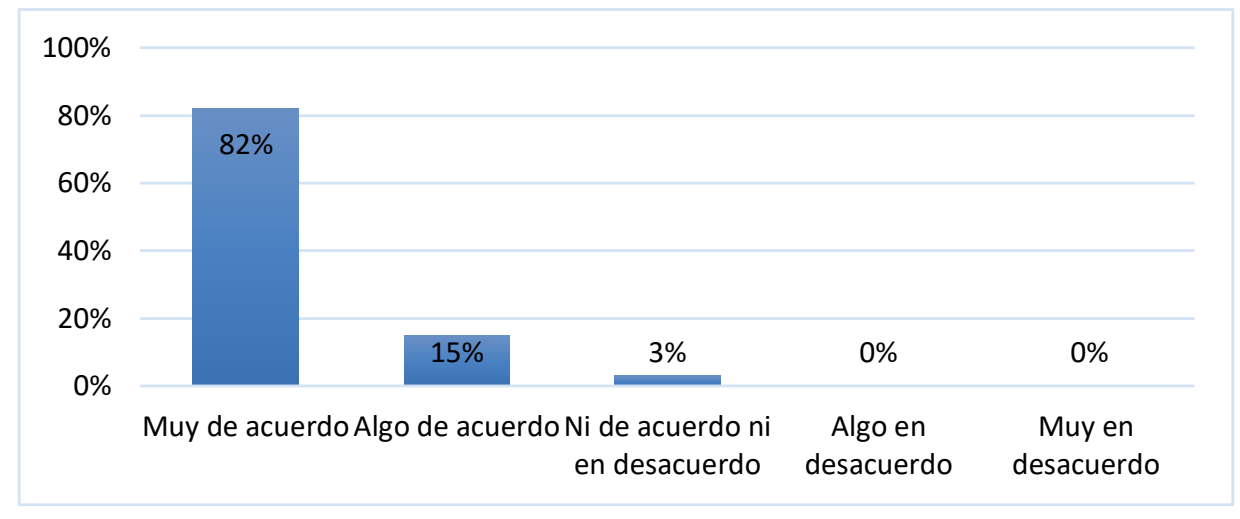

Fuente: Encuestas aplicadas.

\subsection{Me gusta que se realicen este tipo de actividades académicas.}

El 90\% de los estudiantes planteó que le gusta que se realicen este tipo de actividades estando muy de acuerdo con la realización de las mismas. El 10 \% planteó estar algo de acuerdo y aunque es un porcentaje menor cabe encender las alertas porque la actividad investigativa y la presentación de los resultados debe ser centro de interés de los futuros profesionales.

Gráfico 7: Me gusta que se realicen este tipo de actividades académicas.

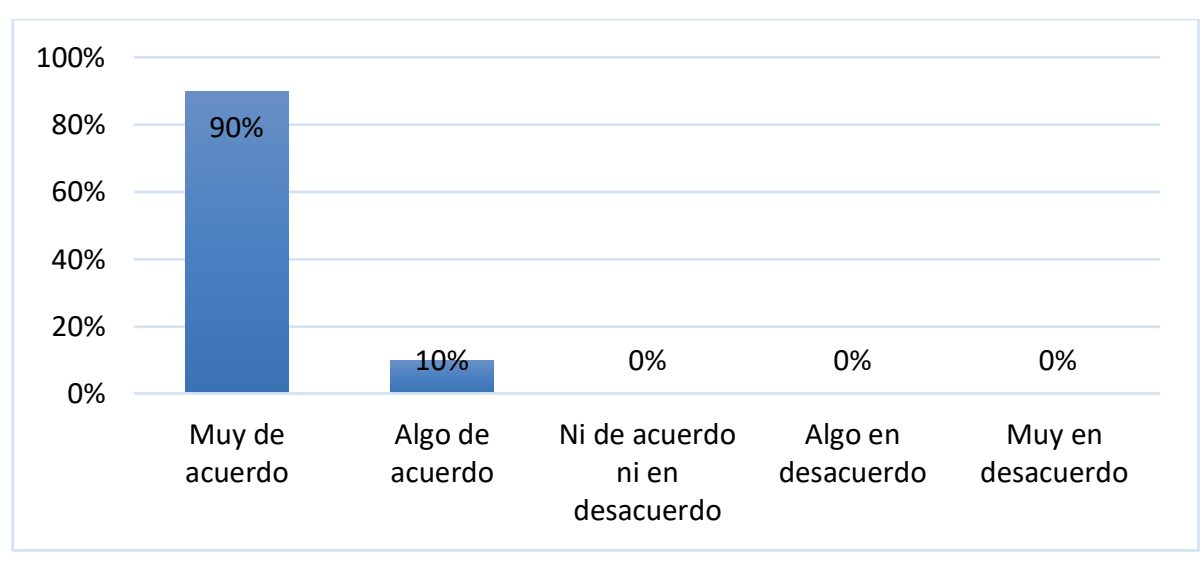

Fuente: Encuestas aplicadas.

\subsection{Las actividades investigativas contribuyen al desarrollo socio económico del país.}

La mayor parte de los estudiantes reconoció como las actividades investigativas contribuyen con los resultados que ofrecen al desarrollo socio económico del país. Se evidencian que el $75 \%$ de los estudiantes opinan estar muy de acuerdo, solo destacar 
que el $10 \%$ considera ni de acuerdo ni en desacuerdo, que siendo un valor inferior se debe trabajar en vías de lograr una cultura investigativa.

Gráfico 8: Las actividades investigativas contribuyen al desarrollo socio económico del país.

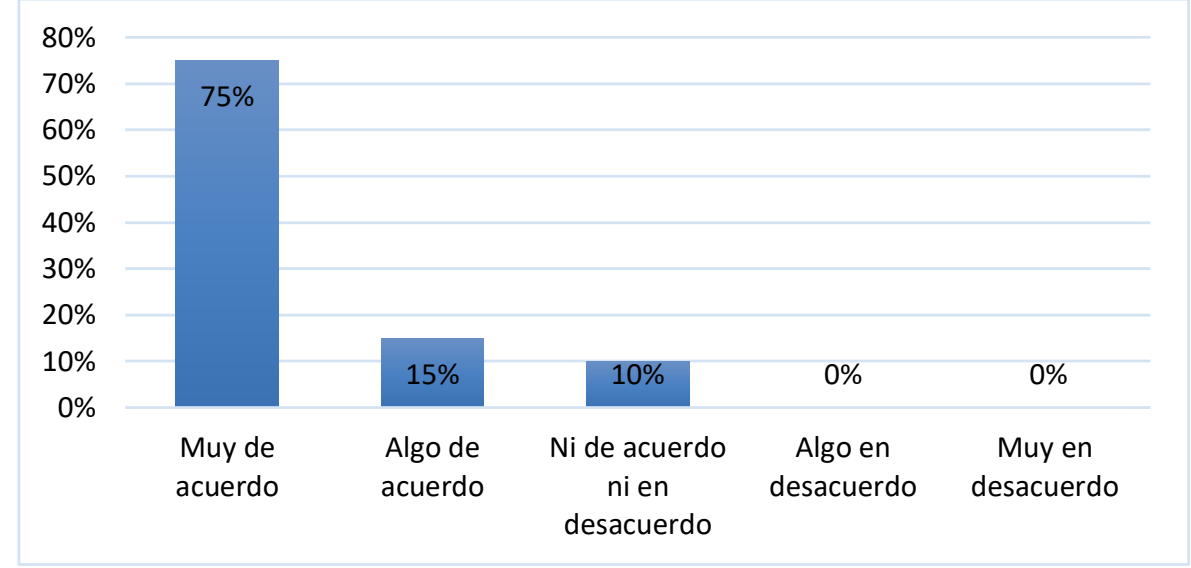

Fuente: Encuestas aplicadas.

3.4 Valoración de la calidad y organización de los trabajos presentados de la cuarta y quinta edición de las Jornadas Científicas Estudiantiles.

La valoración de la calidad y organización de la cuarta y quinta jornada científica fueron evaluadas por lo estudiantes con un alto porcentaje del 95\% lo que demuestra el interés por la investigación y el desarrollo de trabajos investigativos en las diferentes carreras tecnológicas.

Gráfico 9: Valoración de la calidad y organización de los trabajos presentados de la cuarta y quinta edición de las Jornadas Científicas Estudiantiles.

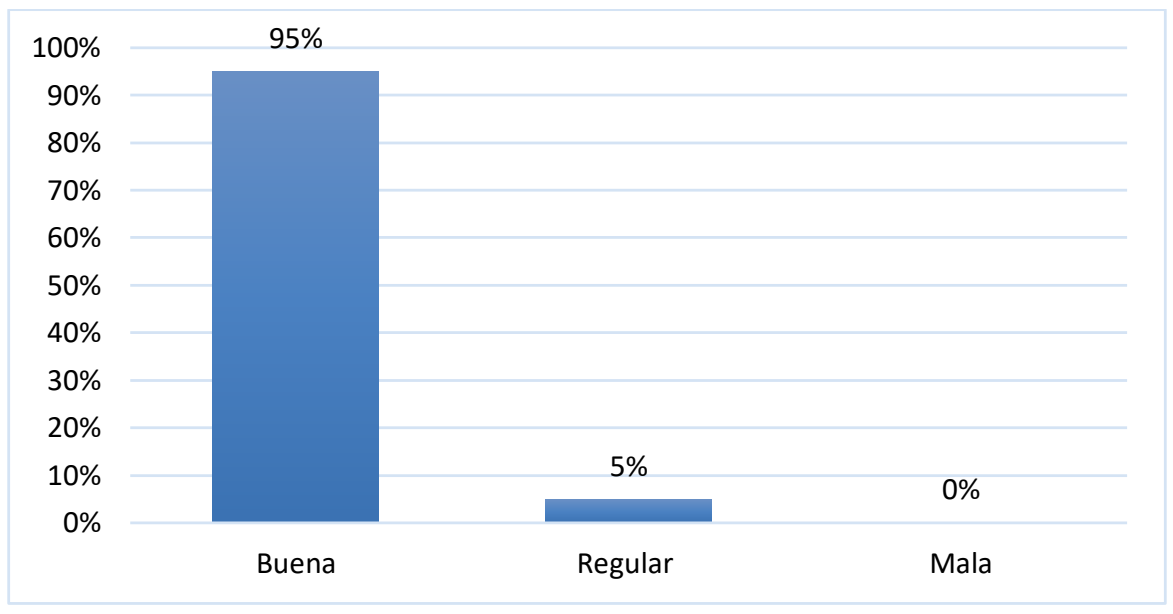

Fuente: Elaboración propia a partir de las encuestas aplicadas. 


\section{CONCLUSIONES}

Es de vital enseñar a investigar teniendo como referente el proceso mismo de la concepción efectiva del conocimiento científico. Los resultados serán mejores basados en la didáctica de la investigación en la enseñanza de las prácticas, procesos, operaciones y mecanismos reales del quehacer científico. Si se quiere enseñar a investigar de una forma práctica es decisivo cambiar el énfasis de la didáctica de la investigación, pasando de una enseñanza teórica, abstracta y general a una didáctica práctica, basada en la capacitación y el entrenamiento en todas y cada una de las operaciones que ocurren real y efectivamente durante el proceso de la producción del conocimiento científico.

El análisis realizado lleva a concluir en que no se enseña a investigar solo desde el aula y con la enseñanza conceptual de la investigación en un enfoque válido, también limita el quehacer científico. Cuando se toman en cuenta estas consideraciones se logran alcanzar los resultados obtenidos por el Instituto Tecnológico de Formación Profesional, Administrativa y Comercial trabajando bajo la perspectiva de un enfoque que enseña a definir, a describir, a analizar, incluso a criticar la producción científica, contribuyendo a la generación de la misma.

La Participación de los estudiantes en las diferentes jornadas científicas ha motivado a la Institución a realizar en el próximo año el I Congreso Internacional de Formación Investigación y aplicaciones Técnico Tecnológicas; y a la creación de una revista estudiantil para la promoción de los resultados científicos de los estudiantes.

\section{BIBLIOGRAFÍA}

Alamillo M. \&Villamor F, (2010). Sobre las regularidades que se presentan en el proceso de enseñanza de la Educación Superior.La Habana: MES.

Baxter, E., (2002). E. Fundamentos teóricos de la dirección del proceso de enseñanza. La Habana: Editorial Pueblo Educación.

Bueno, E. \& Morcillo P. (2002). Enfoques principales de dirección del conocimiento: una síntesis. Boletín Intellectus.

Cadena-Badilla, M., Mejías Acosta, A., Vega-Robles, A., \& Vásquez Quiroga, J. (2015). La satisfacción estudiantil universitaria: análisis estratégico a partir del análisis de factores. Industrial Data, 18(1), 9-18. 
CEPAL/UNESCO, (1992). Educación y conocimiento. Eje de la transformación productiva con equidad. Santiago de Chile: ONU.

Cortés, E., Campos, M., \& Moreno, M. P. (2014). Priorización de las Dimensiones de Evaluación al Desempeño Docente por el Estudiante, en tres Áreas del Conocimiento. Formación universitaria, 7(2), 3-10.

Hernández, R., et al, (2005). Los Enigmas de la educación Superior. México D.F.: Editorial Trillas.

Inciarte, M. (2009). "Diseño Instruccional por Competencias para Administrar Unidades Curriculares Virtualizadas" en Hecademus, Revista Científica de la Fundación Iberoamericana para la Excelencia Educativa, Vol., 2 No. 6.

Le Boterf, (2001). Alternativas para el Desarrollo de Competencias. Barcelona: Editorial Cambria.

Levy-Leboyer, (1996). La Universidad Europea y la Globalización. Madrid: Editorial Santillana.

Ramírez, Z. (1999). La Educación Superior en El Caribe. La Habana: Editorial Félix Varela.

Real Academia Española. Diccionario de la lengua española. (2001). Madrid. (s. e.).

Restrepo, G. (1998). La Educación del Futuro, ¿Cómo Enfrentarla? Bogotá: Editorial Océano.

Rizo, S. (2004). Evaluación de Competencias. Universidad de Nueva Granada, San Sebastián, España.

Sánchez Puentes, R. (2014). Enseñar a investigar Una didáctica nueva de la investigación en ciencias sociales y humanas (Cuarta edición ed.). México: iiSUE.

Tobón, S. et al, (2006). La Formación Basada en Competencias. Bogotá: Ecoe Ediciones.

Torres, M., (2002). Correspondencia Entre Empresas y Universidad. Sevilla: Editorial Casa Blanca.

Touzin, R. (1998). Los Entretelones de la Educación por Competencias. Aragón: Editorial, Castilla.

Zemelman, H. (2009). Los Desafíos de la Educación Superior. Buenos Aires: Editorial Kapeluz. 